\title{
Epidemic Intelligence: For the Crowd, by the Crowd
}

\author{
Avaré Stewart and Ernesto Diaz \\ L3S Research Center, \\ Appelstrasse 4, 30167 Hannover, Germany \\ \{stewart, diaz\}@L3S.de \\ http://www.L3S.de
}

\begin{abstract}
Event Based Epidemic Intelligence (e-EI) encompasses activities related to early warnings and their assessments as part of the outbreak investigation task. Recently, modern disease surveillance systems have started to also monitor social media streams, with the objective of improving their timeliness in detecting disease outbreaks, and producing warnings against potential public health threats.

In this tutorial we show how social media analysis can be exploited for two important stages of e-EI, namely: (i) Early Outbreak Detection, and (ii) Outbreak Analysis and Control. We discuss techniques and methods for detecting health-related events from unstructured text and outline approaches, as well as the challenges faced in social media-based surveillance. In particular, we will show how using Twitter can help us to find early cases of an outbreak, as well as, understand the potential causes of contamination and spread from the perspective of the field practitioners.
\end{abstract}

\section{Introduction}

Social Media streams, such as Twitter and other real-time media are now seen as a valuable source of temporally and spacially relevant information. Applications that rely upon social media streams include trend detection [6, 10]; and intelligence gathering for applications such as: natural disaster detection [7, 9] or flu outbreak tracking [1 13,8$]$.

In this tutorial, we focus on the application of social media streams for EventBased Epidemic Intelligence (e-EI). e-EI encompasses activities related to early warnings and their assessment as part of the outbreak investigation task. The tutorial is divided into two parts, in each, we take up an important aspect of e-EI. In Part I: Early Outbreak Detection, we discuss both supervised and unsupervised techniques for detecting health-related events from unstructured text. We also outline the approaches for detecting relevant disease-reporting entities; such as affected organisms, medical condition, location and temporal mentions and address the problem of disambigating semantic relations which contain these entities. Next we discuss the approaches and challenges of generating early warnings from relevant semantic relations, for domain experts [4]. 
In Part II: Outbreak Analysis and Control, we focus on two types of support for helping domain experts with the problem of information overload when assessing the risk associated with system generated warnings; namely, ranking of early warning alerts [5] and appropriate visualization services. As a case study, we show how using Twitter can help us to find early cases of an outbreak, as well as, understand the potential causes of contamination and spread from the perspective of the field practitioners. Throughout the tutorial, we present practical lessons that have been learned within the context of the M-Eco project (http://www.meco-project.eu/) and numerous results from field practitioners assessments; finally we conclude with an outlook for the domain of e-EI.

Acknowledgments. This work was funded, in part, by the European Commission Seventh Framework Program (FP7/2007-2013) under grant agreement No.247829 for the M-Eco Medical Ecosystem Project.

\section{References}

1. Aramaki, E., Maskawa, S., Morita, M.: Twitter catches the flu: Detecting influenza epidemics using twitter. In: Proceedings of EMNLP 2011 (2011)

2. Collier, N., Son, N.T., Nguyen, N.M.: Omg u got flu? analysis of shared health messages for bio-surveillance. CoRR abs/1110.3089 (2011)

3. Culotta, A.: Towards detecting influenza epidemics by analyzing twitter messages. In: Proceedings of the First Workshop on Social Media Analytics, SOMA 2010 (2010)

4. Diaz, E., Stewart, A.: Tracking twitter for epidemic intelligence. case study: Ehec/hus outbreak in germany. In: ACM Conference on Web Science 2012 (2012)

5. Diaz, E., Stewart, A., Valasco, E., Denecke, K.: Towards personalized learning to rank for epidemic intelligence based on social media streams. In: Proceeding of the World Wide Web 2012 (2012)

6. Mathioudakis, M., Koudas, N.: Twittermonitor: trend detection over the twitter stream. In: Proceedings of SIGMOD 2010 (2010)

7. Sakaki, T., Okazaki, M., Matsuo, Y.: Earthquake shakes twitter users: real-time event detection by social sensors. In: Proceedings of WWW 2010 (2010)

8. Szomszor, M., Kostkova, P., Louis, C.S.: Twitter informatics: Tracking and understanding public reaction during the 2009 swine flu pandemic. In: Proceedings of WI-IAT 2011 (2011)

9. Vieweg, S., Hughes, A.L., Starbird, K., Palen, L.: Microblogging during two natural hazards events: what twitter may contribute to situational awareness. In: Proceedings of CHI 2010 (2010)

10. Yang, J., Leskovec, J.: Patterns of temporal variation in online media. In: Proceedings of WSDM 2011 (2011) 